\title{
PAI-1, fibrosis, and the elusive provisional fibrin matrix
}

\author{
David J. Loskutoff and James P. Quigley
}

Department of Vascular Biology, The Scripps Research Institute, La Jolla, California, USA

Address correspondence to: David J. Loskutoff, Department of Vascular Biology, The Scripps Research Institute, 10550 N. Torrey Pines Road, Maildrop VB-3, La Jolla, California 92037, USA. Phone: (858) 784-7125; Fax: (858) 784-7353; E-mail: loskutof@scripps.edu.

Whether induced surgically or by hypertension, infections, extreme heat, or caustic chemicals, tissue injury invariably leads to vasodilatation, with subsequent leakage of plasma proteins into the connective tissues, rapid activation of the coagulation cascade, and deposition of fibrin. A central paradigm in the field is that the fibrin is organized into a "provisional fibrin matrix," which acts as a road map to direct the migration of invading cells. Leukocytes and possibly fibroblasts migrate into the area and elaborate cytokines which, in turn, stimulate resident cells to synthesize and deposit collagens and other insoluble fibrillar components into the evolving extracellular matrix (ECM).

Fibrotic disease occurs when normal control of this process is compromised and excess fibrous material accumulates in the tissues. It is generally assumed that the persistence of fibrin in the matrix promotes fibrosis, and that the extent of fibrosis is limited by proteinases that remove the fibrin (i.e., the fibrinolytic system). In a recent issue of the JCI, Hattori et al. (1) affirm previous suggestions that plasminogen activator inhibitor-1 (PAI-1) promotes pathological fibrosis but challenges the concept that fibrin is required.

\section{PAI-1 and fibrosis}

The primary fibrinolytic enzyme is plasmin (2), a trypsin-like proteinase that is formed when the zymogen plasminogen is cleaved into its twochain form by either tissue-type plasminogen activator (tPA) or urokinasetype plasminogen activator (uPA). PAI- 1 is the primary inhibitor of both PAs, while $\alpha_{2}$-antiplasmin is the physiological inhibitor of plasmin. Together, these two inhibitors constitute a powerful, negative regulatory system for controlling the formation and activity of plasmin.

Fibrotic disease occurs in most tissues, suggesting that a common path- way mediates this response. Although little is known about specific mechanisms, it is generally assumed that molecules that promote collagen deposition or inhibit its removal must be involved. PAI-1 would seem to be a good candidate for such a molecule, since it is consistently and dramatically upregulated in a variety of fibrotic diseases, including glomerulosclerosis induced by either hypertension or $\mathrm{x}$ irradiation (3), liver fibrosis induced by carbon tetrachloride (4), and spontaneously occurring (5) or bleomycininduced pulmonary fibrosis (6). Importantly, bleomycin-induced fibrosis is more severe in transgenic mice overexpressing PAI-1, and less so in PAI-1 deficient mice (7). Hattori et al. (1) confirm the importance of PAI-1 in this process by demonstrating that fibrinolysis is enhanced, collagen buildup is reduced, and survival is dramatically prolonged in bleomycin-treated PAI-1-deficient mice compared with PAI-1-expressing controls. Taken together, these observations would seem to suggest that the abnormal induction of PAI-1 during tissue repair decreases PA activity and the extent of plasmin formed, thus stabilizing the ECM. There is, however, another possible scenario for these effects, since PAI-1 also has been shown to regulate the adhesion and migration of a variety of cells in vitro and in vivo $(8,9)$. According to this alternative model, the elevated PAI-1 promotes collagen deposition not by inhibiting plasmin, but by stimulating the migration of leukocytes and collagen-producing cells into the damaged tissue. Based on these considerations, it is not clear whether the mechanism of action of PAI-1 depends on its protease inhibitory activity or its effects on cell adhesion and migration. If the former, we assume that the targets of PAI- 1 are uPA and tPA, the major plasmin-generating enzymes, and that the relevant substrate for the plasmin is fibrin, a major component of clots and a protein whose turnover is largely mediated by plasmin. However, this issue is also complicated, since plasmin can activate cytokines and matrix metalloproteinases (MMPs) (10), and these mediators may influence the fibrotic process. Thus, while elevated PAI-1 promotes fibrosis, and decreased PAI1 reduces it, the mechanism of these effects remains elusive.

\section{Fibrinogen-deficient mice}

The article by Hattori et al. (1) also provides important insights into these mechanisms. First of all, this study shows that tranexamic acid, a synthetic inhibitor of both plasmin formation and plasmin activity, reverses the protective effects of PAI- 1 deficiency. That is to say, PAI-1-deficient mice treated with bleomycin in the presence of tranexamic acid develop the same degree of pulmonary fibrosis as do treated wild-type mice. This observation, together with data showing that infusion of uPA into the lungs of these mice also protects against fibrosis (11) and that mice with targeted deletion of the plasminogen gene develop increased fibrosis following lung injury (12), firmly establishes a role for plasmin in both normal and pathological ECM remodeling after injury. Moreover, these observations suggest that deletion of the PAI-1 gene protects against fibrosis by enhancing plasmin formation, not by modulating cell migration. The observation that a similar number of leukocytes is detected in the lungs of bleomycin-treated wild-type and PAI-1 -deficient mice further supports this idea, although the status of the collagen-producing fibroblasts is not discussed in this report.

Hattori et al. (1) next tried to identify the critical substrate for the newly generated plasmin in this fibrotic pathway. They, like others, assumed it 
to be fibrin, because this protein is the best known substrate of plasmin and is chemotactic during wound healing. According to this hypothesis, the increased plasmin activity should accelerate the removal of the fibrin matrix and, as a consequence, decrease the number of infiltrating cells, the degree of collagen disposition, and the magnitude of fibrosis. Indeed, the lungs of bleomycin-treated PAI-1-deficient mice contained less fibrin and more fibrin degradation products than those from similarly treated wildtype mice. Moreover, tranexamic acid restored the fibrin levels in the PAI-1 -deficient mice to those of the wildtype mice. These compelling results confirm that fibrin is a substrate for plasmin. However, as has happened in the past in this field, strong correlations frequently fail to hold up in the face of simple genetics.

The final, remarkable set of results in this report indicates that fibrin is not the key plasmin substrate. In these experiments, Hattori et al. (1) infused bleomycin into the lungs of mice genetically deficient in the gene encoding the A $\alpha$ chain of fibrinogen (13). These mice have no detectable levels of circulating fibrinogen. Unexpectedly, the degree of fibrosis in the bleomycintreated wild-type and fibrinogen-deficient mice was not significantly different. Thus, in spite of the early and consistent presence of fibrin in fibrotic tissue, and despite the fact that fibrin forms a provisional matrix for wound repair, fibrosis still occurs in these fibrinogen-deficient mice.

Do these findings indicate that fibrin does not contribute to the fibrotic process? Probably not, since previous experiments from Degen and colleagues show that although scar tissue develops in the liver (13) and skin (14) of injured $\mathrm{fib}^{-/-}$mice, the process is substantially delayed in both instances. Whether the fibrotic process is also delayed in the bleomycin experiments reported here (1) is not clear from the data presented. In any case, fibrin would seem to promote, but not to be required for, fibrosis.

\section{Other considerations}

Although Hattori et al. (1) provide a number of important insights into mechanisms of fibrosis, their work also raises new questions. Foremost among these is the identity of the biological target of plasmin, since fibrin is not required for fibrosis in this model, and plasmin does not digest collagen or many other fibrillar matrix proteins (10). Thus, the possibility emerges that plasmin's effects are indirect and that plasmin proteolytically activates other regulators of ECM accumulation or degradation. For example, plasmin converts latent TGF- $\beta$ into the active form, and active TGF- $\beta$, among its many other effects, stimulates ECM production by fibroblasts (15). However, this scenario seems unlikely, since the increased plasmin in PAI-1-deficient mice is associated with diminished fibrosis, and plasmin-activated TGF- $\beta$ would be expected to promote fibrosis $(3,15)$. Plasmin also can activate MMPs, enzymes that can degrade most ECM proteins including collagen, the major proteinaceous component of fibrotic tissue (10). In this regard, it is important to point out that plasmin is often cited as a general activator of MMPs and therefore often implicated as a crossover protease between serine protease cascades and MMPmediated pathways. However, plasmin efficiently activates only MMP-1 (collagenase-1) and MMP-3 (stromelysin-1) (16). It does not directly activate a number of other important MMPs, including MMP-2 (gelatinase A) and MMP-9 (gelatinase B) (17). The ability of plasmin to activate additional MMP zymogens remains to be tested rigorously. If, however, specific MMPs can be shown to protect against fibrosis, then inhibitors (e.g., tissue inhibitors of MMPs or tissue inhibitors of matrix metalloproteinases (TIMPs), as well as zinc chelators and synthetic inhibitors) might mimic tranexamic acid and promote fibrosis, even in the PAI-1 -deficient mouse. Interestingly, TIMP expression not only is elevated in fibrotic disease, but also is frequently under the control of the same molecules that regulate PAI-1 expression (3, 10). Thus, if TIMPs can be implicated in the fibrotic response to injury, one would predict that they may be induced by agents or conditions that induce fibrosis, and that TIMP knockout mice might be protected from this condition. A TIMP $P^{-/-P A I-1^{-/-}}$double knockout mouse might be completely protected against fibrotic disease. Whatever the mechanism, if plasmin controls the degree of fibrosis, as seems likely from the studies of Hattori et al.
(1), then it is possible that $\alpha_{2}$-antiplasmin, which has been detected in a variety of tissues (18), may also contribute to this process. Thus, transgenic mice overexpressing $\alpha_{2}$-antiplasmin and $\alpha_{2^{-}}$ antiplasmin-deficient mice also should be examined for changes in fibrosis in response to bleomycin.

Finally, a few additional caveats are in order. PAI-1 inhibits thrombin and other proteases $(19,20)$, and these, in turn, may also influence the fibrotic response to injury. In addition, plasmin-mediated fibrin degradation products have long been known to be biologically active, and to stimulate a variety of cellular activities, including cell migration and PAI-1 gene expression (21). Although the fibrinogendeficient mice employed by Hattori et al. (1) lacked the gene for the A $\alpha$ chain of fibrinogen, the genes for the other two chains of fibrinogen (B $\beta$ and $\gamma$ ) presumably are still expressed in these mice. Thus, although it is unlikely, it is possible that plasmin-digested fragments generated from these chains are present in the damaged lung tissue and also influence the fibrotic process.

In summary, as seen in the studies of Hattori et al. (1) and others (7, 11-13), components of the fibrinolytic system play a central regulatory role in pulmonary fibrosis. Analysis of $P A I-1^{-/-}, \mathrm{fib}^{-/-}, \mathrm{plg}^{-/-}, \mathrm{uPA^{-/- }}$, and other genetically deficient mice has offered some insights into the relative contribution of the individual components of this system. Unraveling the interactions among these components in bleomycin-induced fibrosis may require the study of animals with multiple genetic deficiencies, such as

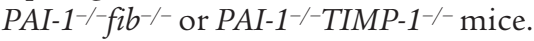
Some of these studies already appear to be underway (1). Whatever the details, it is quite clear that a critical regulatory role in fibrosis is played by PAI-1. Hattori et al. (1) indicate that it is the antiproteolytic activity of PAI-1, and not its effects on cell migration, that is important in this disease process. While their argument is compelling, this issue has not yet been rigorously demonstrated. In this regard, genetic or viral delivery of PAI-1 to sites of fibrosis in PAI-1-/- mice would be a powerful means to define the mechanism more precisely. If wildtype PAI-1 delivered to the lungs of PAI-1 $1^{-/}$mice overcomes the resistance to fibrosis, then select mutants of PAI- 
1 that either are noninhibitory or no longer bind vitronectin can be similarly delivered. Such studies should distinguish which of PAI-1's biochemical features control fibrosis.

1. Hattori, N., et al. 2000. Bleomycin-induced pulmonary fibrosis in fibrinogen-null mice. J. Clin. Invest. 106:1341-1350.

2. Hekman, C.M., and Loskutoff, D.J. 1987. Fibrinolytic pathways and the endothelium. Semin. Thromb. Hemost. 13:514-527.

3. Fogo, A.B. 1999. Mesangial matrix modulation and glomerulosclerosis. Exp. Nephrol. 7:147-159.

4. Zhang, L.P., et al. 1999. Increased expression of plasminogen activator and plasminogen activator inhibitor during liver fibrogenesis of rats: role of stellate cells. J. Hepatol. 31:703-711.

5. Eitzman, D.T., and Ginsburg, D. 1997. Of mice and men. The function of plasminogen activator inhibitors (PAIs) in vivo. Adv. Exp. Med. Biol. 425:131-141.

6. Olman, M.A., Mackman, N., Gladson, C.L., Moser, K.M., and Loskutoff, D.J. 1995. Changes in procoagulant and fibrinolytic gene expression during bleomycin-induced lung injury in the mouse. J. Clin. Invest. 96:1621-1630.

7. Eitzman, D.T., et al. 1996. Bleomycin-induced pulmonary fibrosis in transgenic mice that either lack or overexpress the murine plasminogen activator inhibitor-1 gene. J. Clin Invest. 97:232-237.

8. Chapman, H.A. 1997. Plasminogen activators integrins, and the coordinated regulation of cell adhesion and migration. Curr. Opin. Cell Biol. 9:714-724.

9. Bajou, K., et al. 1998. Absence of host plasminogen activator inhibitor 1 prevents cancer invasion and vascularization. Nat. Med. 4:923-928.

10. Parks, W.C., and Mecham, R.P. 1998. Matrix metalloproteinases. Academic Press. San Diego, California, USA. 1-362.

11. Sisson, T.H., Hattori, N., Xu, Y., and Simon, R.H. 1999. Treatment of bleomycin-induced pulmonary fibrosis by transfer of urokinasetype plasminogen activator genes. Hum. Gene Ther. 10:2315-2323.

12. Swaisgood, C.M., French, E.L., Noga, C., Simon, R.H., and Ploplis, V.A. 2000. The development of bleomycin-induced pulmonary fibrosis in mice deficient for components of the fibrinolytic system. Am. J. Pathol. 157:177-187.

13. Suh, T.T., et al. 1995. Resolution of spontaneous bleeding events but failure of pregnancy in fibrinogen-deficient mice. Genes Dev. 9:2020-2033.

14. Bugge, T.H., et al. 1996. Loss of fibrinogen rescues mice from the pleiotropic effects of plas- minogen deficiency. Cell. 87:709-719.

15. Gleizes, P.-E., et al. 1997. TGF- $\beta$ latency: biological significance and mechanisms of activation. Stem Cells. 15:190-197.

16. Murphy, G., et al. 1999. Mechanisms for pro matrix metalloproteinase activation. APMIS 107:38-44.

17. Ramos-DeSimone, N., Hahn-Dantona, E., Sipley, J., Nagase, H., and Quigley, J.P. 1999. Activation of matrix metalloproteinase-9 (MMP-9) via a converging plasmin/stromelysin- 1 cascade enhances tumor cell invasion. J. Biol. Chem. 274:13066-13076

18. Menoud, P.-A., Sappino, N., Boudal-Khoshbeen, M., Vassalli, J.-D., and Sappino, A.-P. 1996. The kidney is a major site of $\alpha_{2}$-antiplasmin production. J. Clin. Invest. 97:2478-2484

19. Hekman, C.M., and Loskutoff, D.J. 1988. Bovine plasminogen activator inhibitor 1 : specificity determinations and comparison of the active, latent and guanidine-activated forms. Biochemistry. 27:2911-2918.

20. van Meijer, M., and Pannekoek, H. 1995. Structure of plasminogen activator inhibitor 1 (PAI1 ) and its function in fibrinolysis: an update. Fibrinolysis. 9:263-276.

21. Olman, M.A., et al. 1999. Fibrin fragment induction of plasminogen activator inhibitor transcription is mediated by activator protein 1 through a highly conserved element. Blood. 6:2029-2038 